\title{
Hydrocolloid-based nutraceutical delivery systems: Effect of counter-ions on the encapsulation and release
}

\author{
Patrick J. Polowsky ${ }^{\mathrm{a}}$, Srinivas Janaswamy ${ }^{\mathrm{a}, \mathrm{b}^{*}}$
}

${ }^{\mathrm{a}}$ Department of Food Science, ${ }^{\mathrm{b}}$ Whistler Center for Carbohydrate Research, Department of Food Science, Purdue University, West Lafayette, IN 47907-2009, USA

${ }^{*}$ Corresponding author

745 Agriculture Mall Drive, Purdue University, West Lafayette, IN 47907-2009, USA

Tel: (765) 494-4914

Fax: (765) 494-7953

E-mail: janaswam@purdue.edu

May 9, 2014

Submitted to

Food Hydrocolloids

Revision

July 25, 2012 


\begin{abstract}
Nutraceuticals provide health benefits, especially for the prevention and treatment of chronic diseases such as diabetes, obesity, cardiovascular disease and cancer. Their incorporation in food supplements, functional foods and medicinal foods is a major technological challenge due to lower water solubility, instability during processing and storage conditions. Carriers that can effectively overcome these predicaments and protect them during product development, consumption and delivery are in high demand. Toward this end, our research approach is to entrap nutraceuticals in the ordered networks of hydrocolloids. We have examined the effect cations in regulating the encapsulated amounts and release characteristics. Iota-carrageenan and eugenol have been chosen as models of hydrocolloid and nutraceutical, respectively, in the presence of $\mathrm{Na}$ and $\mathrm{Ca}$ ions. The results suggest that carrageenan maintains its network organization even after encapsulating the eugenol molecules. Increased eugenol amounts are found in the Na carrageenan complex compared to the Ca complex, and the release rate is faster from the former but it is more controlled from the latter. These differences highlight the vital role of cations on the encapsulation efficiency and release profiles of hydrocolloid-based nutraceutical carriers. The outcome offers an elegant opportunity for developing novel and value-added food systems employing low-in-cost, nontoxic and heavily consumed food grade hydrocolloids.
\end{abstract}




\section{Introduction}

Nutraceuticals are beneficial due to their inherent health advantages and thus are suited well for developing novel food supplements, functional foods and medicinal foods (Goldberg, 1994;

Webb, 2006). They are sparingly water soluble, however, and somewhat unstable to environmental conditions such as temperature, light, and oxygen (Bell, 2001). As their bioavailability greatly controls the overall efficacy, carriers play significant role in developing effective nutraceutical products. The general purpose of carriers, in this regard, is to protect the encapsulated molecules from external stressors, preserve functionality and deliver efficaciously at the target site. Synthetic polymer networks (Langer, 1990; Langer \& Tirrell, 2004), protein matrices (Chen, Remondetto \& Subirade, 2006), and nanoparticles (Acosta, 2009; Plapied, Duhem, des Rieux \& Préat, 2011), to name a few, are known to be efficient for entrapment and release but their non-rigid assemblies and the ensuing incompatibilities to the human digestive system are major challenges to the optimization of ideal nutraceutical delivery systems. In our ongoing efforts towards the design and development of carriers based on human compatible biopolymers possessing stable architectures that can satisfactorily retain functionality, we successfully demonstrated the feasibility of hydrocolloid fibers for the controlled release of nutraceuticals (Janaswamy \& Youngren, 2012) and drugs (Janaswamy, Gill, Campanella \& Pinal, 2013). Our research on anionic iota-carrageenan, sulfated hydrocolloid from marine algae with widespread utility in food, non-food, pharmaceutical and medical applications (Stanley, 1990), fibers reveal that upon encapsulation, guest molecules tightly cradled in the water pockets of carrageenan network are protected from heat and released in sustained manner. Furthermore, the van der Waals, hydrogen bonding and electrostatic interactions among the embedded molecules, carrageenan helices and the charge balancing cations appear to control the release 
characteristics. This important observation forms the basis for the present investigation. Herein, we compared the influence of mono- and di-valent cations on the encapsulation efficiency and release behavior of nutraceuticals by studying the Na and Ca salt forms of iota-carrageenan (IC) and eugenol as the model hydrocolloid and model nutraceutical, respectively.

Eugenol is a phenolic compound (1-hydroxy-2-methoxy-4-allylbenzene) generally derived from clove (Lee \& Shibamoto, 2001), basil (Raina, Kumar, \& Dutta, 2013), and cinnamon (Dighe, Gursale, Sane, Menon \& Patel, 2005). It gained interest in the medical field as an antimicrobial, antiviral and antioxidant agent (Benencia \& Courreges, 2000; Palaniappan \& Holley, 2010). Eugenol can suppress inflammatory response and tumor growth, specifically in the expression of cyclooxygenase-2 by playing a key role in the reduction of carcinogenesis (Jaganathan, Mazumdar, Mondhe \& Mandal, 2011; Kim et al., 2003). It has the ability to denature proteins as well as change cell permeability by reacting with the membrane phospholipids (Briozzo et al., 1989). Eugenol is also used in the treatment of periodontal diseases (Jadhav, Khandelwal, Ketkar, \& Pisal, 2004), and in certain food applications. Despite several benefits, its sparingly water-soluble nature coupled with instability at room temperature and photosensitive character limits eugenol wholesome utility.

Our results reveal that Na network has higher loading amounts of eugenol and faster release rates while Ca network accommodates lesser quantities but is able to release them in a more controlled manner; highlighting the indispensable role of cations in tailoring the functional behavior of hydrocolloid-based nutraceutical carriers. 


\section{Materials and Methods}

\subsection{Materials}

Research grade IC sample (RE-PR-4018) was provided by FMC Corporation, USA. NaCl (X190-1Kg, Lot \# 1502C144), CaCl (BDH4524-00GP, Lot \# YH0231NFBD) and eugenol (A14332, Lot \# 10172314) were purchased from Amresco, VWR International and Alfa Aesar, respectively. Reagent grade isopropyl alcohol and double distilled water were used as necessary.

\subsection{Sodium and calcium salt form of IC}

IC was converted into pure sodium and calcium salt forms as follows: $400 \mathrm{mg}$ of IC was dissolved in $100 \mathrm{mM}$ salt $\left(\mathrm{NaCl}\right.$ or $\left.\mathrm{CaCl}_{2}\right)$ solution. The solution was heated to and held at $90{ }^{\circ} \mathrm{C}$ with constant stirring for about 45 minutes. The hot mixture was then poured into two volumes of cold isopropanol $\left(0^{\circ} \mathrm{C}\right)$ to precipitate out the hydrocolloid. The coagulum then was separated from the solution and rinsed thoroughly in 80 and 100\% isopropanol to remove any unbound cations. The final product was dried at $50{ }^{\circ} \mathrm{C}$ for a day.

\subsection{Fiber preparation}

The experimental approach to engineer IC fibers and load nutraceutical molecules in the organized network is the novelty of our research. This, in part, requires well-organized fiber formation, orientation and crystallinity. Fig. 1 highlights the experimental procedure. Briefly, $10 \mathrm{mg}$ of IC (two separate vials for sodium and calcium forms) was dissolved in $1 \mathrm{~mL}$ of deionized distilled water in a vial. Homogeneous solution was obtained by heating the vial at $90{ }^{\circ} \mathrm{C}$ for 30 minutes with intermediate vortexing, and later cooled to room temperature. A $20 \mu \mathrm{L}$ droplet was placed in between two glass rods in a fiber puller at $66 \%$ relative humidity (RH). 
Once the droplet dried partially (after around 3 - 4 hrs), it was stretched to about 2 - $3 \mathrm{~mm}$ in length at regular intervals, and was allowed to dry further for 12 hrs in the fiber puller. Then the fiber was cut loose from the glass rods and stored in a desiccator at 66\% RH for further analysis. Using this protocol, around 40-50 fibers were prepared in a day for a total of 200 fibers from each salt form of IC.

\subsection{IC:eugenol complexes}

The solution prepared contained $18 \mathrm{mg}$ of eugenol dissolved in $19 \mathrm{~mL}$ of isopropanol along with $1 \mathrm{~mL}$ of distilled water. The water addition was for loosening the fiber network during the encapsulation process. Stretched fibers were immersed in the solution for 3 days at temperatures $4,25,35$, and $45^{\circ} \mathrm{C}$. The complex fibers were then removed from the solution and equilibrated at $66 \%$ RH for further analysis.

\subsection{Diffraction patterns and Unit cell dimensions}

Synchrotron X-ray diffraction data were obtained at 14-BMC beamline, BioCARS, Argonne National Laboratory (ANL), Chicago, IL. The wavelength of the X-ray beam was $0.979 \AA$ and each exposure lasted for 5 seconds. Calcite powder (3.035 $\AA$ characteristic spacing) was used for internal calibration. The pattern center, detector to fiber distance, fiber tilt and rotation, unit cell dimensions and Miller indices $(h, k, l)$ for reflections were estimated using FibreFix (Rajkumar, Al-Khayat, Eakins, Knupp \& Squire, 2007) from CCP13 suite of programs. 


\section{6. $\quad$ Melting behavior of IC:eugenol complexes}

Modulated Differential Scanning Calorimetry (mDSC) was performed using a DSC Q2000 from TA instruments (New Castle, DE) on all the IC:eugenol complexes along with the pure eugenol, Na IC and Ca IC fibers. The equipment was calibrated with an Indium disk. Sample sizes of $1.0 \pm 0.1 \mathrm{mg}$ were sealed in Tzero aluminum hermetic pans and were analyzed under a nitrogen gas flow of $50 \mathrm{~mL} / \mathrm{min}$. A modulation of $\pm 0.68^{\circ} \mathrm{C}$ for every minute was used and the temperature was ramped from $0-230^{\circ} \mathrm{C}$ at a constant rate of $3{ }^{\circ} \mathrm{C}$ per minute.

\subsection{Eugenol release from the complexes and encapsulated amount}

Concentration testing was performed using a Beckman Coulter DU 730 Life Science UV/Vis spectrophotometer with disposable UV cuvettes. Initially, wavelength scan was performed in the range 200 to $600 \mathrm{~nm}$ for determining the optimum wavelength of absorbance $(276 \mathrm{~nm})$ for eugenol. Subsequently, calibration curve was generated by dissolving known amounts of eugenol in isopropyl alcohol. Distilled deionized water was used as solvent for estimating the eugenol content in the complex. IC:eugenol fibers weighted around $1.0 \mathrm{mg}$ were placed at the bottom of the cuvette containing $600 \mu \mathrm{L}$ of distilled deionized water. The spectrometer was zeroed before the measurements with cuvette containing only water. The kinetic/time program was used to take continuous measurements in two minute increments for a total of $2.5 \mathrm{hrs}$. Eugenol amounts in the complexes were estimated using the calibration curve with the known absorbances. 


\section{Results and Discussion}

The research strategy was to encapsulate eugenol molecules in the organized networks of monovalent $\left(\mathrm{Na}^{+}\right)$and divalent $\left(\mathrm{Ca}^{2+}\right)$ salt forms of IC so as to understand the influence of cations on the loading amounts and release profiles. The experiments were performed at 4,25 , 35, and $45{ }^{\circ} \mathrm{C}$ for studying the effect of complexing temperature as well. Like any other polysaccharide sample, IC was amorphous but an ordered network was obtained by stretching fibers from $1 \%$ solution. The structural organization has been assessed through X-ray fiber diffraction analysis.

\subsection{Structural characterization}

The diffraction patterns of Na IC and its complexes with eugenol are shown in Fig. 2a-e. The uniformly sharp Bragg reflections in all the four quadrants confirm the long-range ordering in the fibers. In the case of sodium IC (Fig. 2a), the diffraction extends up to $2.76 \AA$ with 15 wellresolved reflections on zero through four layer lines. These are indexable on a trigonal unit cell with dimensions $a=24.1$ and $c=13.0 \AA$, similar to those reported for sodium IC (Janaswamy \& Chandrasekaran, 2001). Eugenol encapsulation has induced substantial changes in the diffraction pattern of IC. For example, the complexes prepared at $4{ }^{\circ} \mathrm{C}$ diffract up to $2.12 \AA$ with 34 reflections spread across the 6 layer lines. While sharp and crystalline Bragg reflections are characteristic of complexes prepared at 4 and $25{ }^{\circ} \mathrm{C}$, slightly diffused and broader are those for the 35 and $45{ }^{\circ} \mathrm{C}$ samples. In all the patterns, the first meridional reflection is on the $3^{\text {rd }}$ layer line establishing that the 3-fold helix of IC is preserved even after eugenol is encapsulated in the carrageenan lattice. However, size and intensity of Bragg reflections on the individual layer lines and changes in their positions suggest alternate packing arrangements. In order to quantify the 
pertinent differences, intensity distribution on the first layer line in the four complexes along with IC are compared in Fig. 3. In IC, there are four major reflections at $\xi=0.0519,0.1435$, 0.1922 and $0.2315 \AA^{-1}$. Interestingly, in the $4{ }^{\circ} \mathrm{C}$ complex, a new reflection appears at very low $\xi$ of $0.0219 \AA^{-1}$ followed by those at $0.0529,0.0732,0.0930,0.1443,0.1716,0.1943,0.2385$ and $0.2625 \AA^{-1}$. Consequently, much larger trigonal basal net with $a=56.5 \AA$ is obtained. On the other hand, in the $25^{\circ} \mathrm{C}$ complex, there are 6 reflections at $0.0260,0.0484,0.0897,0.1393$, 0.1914 and $0.2236 \AA^{-1}$ that yield an orthorhombic net of $a=27.0$ and $b=39.5 \AA$. Those prepared at 35 and $45{ }^{\circ} \mathrm{C}$ prefer intermediate trigonal nets with $a=38.5$ and $38.0 \AA$, respectively. In all of them, the 13.0 A layer line spacing is preserved compatible with the unperturbed molecular structure of IC (half of the helix pitch $=c$ ). The variations in the basal net dimensions and shape unequivocally point out the alterations in the packing arrangements in the complexes. Therefore, the number of eugenol molecules and IC helices involved in each network formation are not the same.

The diffraction patterns from Ca IC and its complexes with eugenol are shown in Fig. 2f-j. There are 16 Bragg reflections in Ca IC that diffract up to $2.57 \AA$ on zero through four layer lines. Compared to Na IC, these reflections are sharper but their arc lengths increase gradually toward the pattern edge due to the formation of larger crystallites with lesser alignment along the fiber axis. The unit cell is trigonal with $a=23.7$ and $c=13.2 \AA$, and is in accord with the reported Ca IC values (Janaswamy \& Chandrasekaran, 2002). Interestingly, this arrangement prevails even after eugenol encapsulation, unlike the Na IC, but with minimal changes found in $a$ as 23.7, 23.4, 23.4 and $23.2 \AA$ for the $4,25,35$ and $45^{\circ} \mathrm{C}$ complexes, respectively. 
Our experiments with guanidinium IC suggest that the unit cell constants are not changed in the temperature range 5 to $70{ }^{\circ} \mathrm{C}$ (unpublished results). We believe this trend will hold good for other cations such as sodium, calcium and iron, and thus analysis about the effect of temperature on the unit cell parameters of Na IC and Ca IC has not been attempted. Overall, X-ray results reveal that the basal net of $\mathrm{Na}$ IC is flexible during eugenol encapsulation; however, the Ca IC network is fairly rigid. The pertinent details of IC:eugenol tertiary structure and their mode of association, especially the cation-eugenol-carrageenan interactions, require further analysis.

\subsection{Thermal protection of eugenol by the hydrocolloid network}

To assess the effect of encapsulation as well as the role of charge balancing cation on the heat stability of eugenol, IC fibers, IC-eugenol complexes and pure eugenol have been subjected to mDSC analysis. The Na IC displays crystallization at around $151{ }^{\circ} \mathrm{C}$ followed by melting at $158{ }^{\circ} \mathrm{C}$ (Fig. 4a). On the other hand, in the case of Ca IC these two events occur at 158 and $170{ }^{\circ} \mathrm{C}$, respectively (Fig. 4b). In the hydrocolloid fibers, both amorphous and crystalline domains coexist. Thus, the observed exothermic transition is due to the crystallization of amorphous content and the endothermic event corresponds to the melting of crystallites. Interestingly, the observed increase in the crystallization and melting temperatures by around 7 and $12{ }^{\circ} \mathrm{C}$, respectively in the Ca IC reflects its stronger inter-helical interactions compared to the Na IC. In both cases, the melting enthalpies are too small to measure, which could be due the presence of sturdy crystalline domains that resist melting. The possibility of melting beyond $230{ }^{\circ} \mathrm{C}$ might not be possible as fibers start to decompose at the end of the experiment, nevertheless measurements at higher temperatures shed light on the melting behavior of IC fibers. 
The thermograms from the complexes clearly demonstration the heat stability of eugenol molecules after encapsulating them in the IC lattice. Eugenol boils at around $212^{\circ} \mathrm{C}$ (Fig. 4c); however, in the Na IC-eugenol complex (Fig. 4d) or Ca IC-eugenol (Fig. 4e) no peak around this temperature was observed. Instead, in Na IC-eugenol one exothermic event occurs at $151{ }^{\circ} \mathrm{C}$ followed by an endothermic transition at $167^{\circ} \mathrm{C}$. Similarly, in Ca IC-eugenol the exothermic peak is at 156 followed by melting at $160{ }^{\circ} \mathrm{C}$. The comparison of Ca IC-eugenol (Fig. 4e) and Na IC-eugenol (Fig. 4d) profiles suggests that presence of eugenol molecules in the network destabilizes the crystalline domains that were more pronounced in the latter network.

In the complexes, absence of eugenol boiling suggests non-existence of inter-molecular interactions between the eugenol molecules. Though the exact number of eugenol molecules embedded in each water pocket will only be revealed after completing the three-dimensional structures analysis of the complexes, there is a possibility for one eugenol molecule to reside. As the successive eugenol pockets are separated laterally by the IC helices (Fig. 1f and 1g) and vertically by two sugar units (chemical repeat of IC) direct eugenol-eugenol interactions are too weak to promote the necessary associations for triggering melting during the DSC heating. The absence of eugenol boiling in the complexes is another compelling evidence that eugenol molecules are cradled in the carrageenan network. We strongly believe that these fiber complexes will aid in retaining the eugenol functionality even after exposing them to light as well as other external stressors and its bioavailability will be preserved for longer durations especially to take advantage of its antimicrobial properties towards addressing the food safety and food spoilage issues. 


\subsection{Eugenol release from the hydrocolloid network}

Fig. 5a depicts the time-dependent release profiles of eugenol from Na and Ca IC fiber complexes prepared at $25^{\circ} \mathrm{C}$. The results clearly showcase the intrinsic ability of cation type in regulating the encapsulated amounts as well as subsequent release profiles. In the case of Na IC, most of the release is over within the initial 20 mins at a faster pace that later slows down and saturates. The load is about $3.2 \mu \mathrm{g}$ of eugenol per mg of IC, measured at $2.5 \mathrm{hrs}$. On the other hand, Ca IC fibers do not release any eugenol during the first 18 mins. Afterwards, the release is gradual and slowly peaks at 36 mins with subsequent saturation. Surprisingly, the load is only $1.6 \mu \mathrm{g} / \mathrm{mg}, 50 \%$ less than the Na IC.

The reason behind distinct encapsulated amounts and release rates can be better understood by analyzing the inter-helical interactions of IC in the presence of $\mathrm{Na}$ and $\mathrm{Ca}$ ions, respectively. IC has a disaccharide chemical repeat of $\rightarrow 3)-\beta$-D-Gal-4- $\mathrm{SO}_{3}{ }^{-}-(1 \rightarrow 4)-3,6$-anhydro- $\alpha$-D-Gal-2- $\mathrm{SO}_{3}{ }^{-}$ $-(1 \rightarrow$. Its three-dimensional structure (Janaswamy \& Chandrasekaran, 2001) reveals that in the presence of Na ions, a pair of helices communicate via a series of hydrogen bonding $(\cdots)$ interactions such as $4-\mathrm{S} \cdots W / \mathrm{Na} \cdots 4-\mathrm{S}, 2-\mathrm{S} \cdots W \cdots W \cdots W \cdots 4-\mathrm{S}, 2-\mathrm{S} \cdots \mathrm{Na} \cdots 2-\mathrm{S}, 2-\mathrm{S} \cdots \mathrm{Na} \cdots 4$-S, 2-S $\cdots$ Na $\cdots W \cdots 2-S$, 4-S $\cdots W \cdots W \cdots 4-S$, 4-S $\cdots$ Na $\cdots W \cdots W \cdots W \cdots 4-S, 2-S \cdots W \cdots N a \cdots 4-S$, 4-S $\cdots \mathrm{Na} \cdots W \cdots \mathrm{Na} \cdots 4$-S, and 2-S $\cdots W \cdots W \cdots W \cdots 4$-S, wherein 4-S, 2-S and $W$ represent 4sulfate group on $\beta$-D-Gal, 2-sulfate group on $\alpha$-D-Gal and water molecule, respectively. On the other hand, direct interactions such as 2-S $\cdots$ Ca $\cdots 2-S$, 2-S $\cdots$ Ca $\cdots 4$-S and 4-S $\cdots$ Ca $\cdots 4$-S are made possible by the Ca ions (Janaswamy \& Chandrasekaran, 2002). Thus, each Ca ion not only balances the charge on two sulfate groups of adjacent helices but also draws them closer and tighter. Whereas, a Na ion merely binds to one sulfate moiety on one helix for charge balance, 
and hence the resulting weaker inter-helical interactions are mediated by space fillers such as water molecules and are propagated laterally. The resulting Na IC network is much more flexible than that of Ca IC. The variations in the corresponding network strengths reflect significantly on the encapsulated amounts and subsequent release profiles. The Na IC lattice relaxes sufficiently enough in accommodating relatively higher amounts of eugenol compared to the rigid Ca IC. Similarly, during the release process, Na IC network dissociates much faster than the Ca IC resulting in the observed quick and delayed releases, respectively.

The eugenol release profile from the Na IC compares well with those reported from the IC:curcumin (Janaswamy \& Youngren, 2012) and IC:griseofulvin (Janaswamy, Gill, Campanella \& Pinal, 2013) complexes. However, the former encapsulates higher amounts of $3.2 \mu \mathrm{g} / \mathrm{mg}$ compared to far less $0.15 \mu \mathrm{g} / \mathrm{mg}$ and $1.8 \mu \mathrm{g} / \mathrm{mg}$, respectively, from the latter two examples. Thus, the guest molecule's chemical structure, size and available functional groups along with their associative kinetics with the carrageenan helices are the dictating factors for the embedding amounts as well as the release characteristics.

Apart from cation type, complexation temperature also governs the encapsulated amounts. For example, in the case of $\mathrm{Na}$ IC, around 3.75, 3.5, $2.5 \mu \mathrm{g} / \mathrm{mg}$ of eugenol can be loaded in the complexes prepared at 45,35 and $4{ }^{\circ} \mathrm{C}$, respectively (Fig, $\mathbf{5 b}$ ). In contrast, the loading amounts are reduced to 2.4, 2.0 and $1.4 \mu \mathrm{g} / \mathrm{mg}$ for Ca IC, in that order (Fig. 5b). At higher temperatures more ordered domains seem to be created in the carrageenan network leading to increased number of water pockets that in-turn augment the eugenol accumulation in the fibers. In the case of IC:curcumin (Janaswamy \& Youngren, 2012), interestingly, $45^{\circ} \mathrm{C}$ complexes hold lesser 
$(0.09 \mu \mathrm{g} / \mathrm{mg})$ quantity compared to $25^{\circ} \mathrm{C}(0.15 \mu \mathrm{g} / \mathrm{mg})$ ones. Thus, the intimate interactions between the guest molecules and hydrocolloid helices are the major influencers in tailoring the encapsulation efficiency and release nature of eugenol in particular and nutraceuticals in general.

\section{Conclusions}

Functional foods and medicinal foods are helpful in regulating the physiological processes, particularly for gaining the overall health benefits (Day, Seymour, Pitts, Konczak, \& Lundin, 2009; Shahidi, 2009). Towards this end, encapsulation and delivery of health promoting and disease preventing compounds through biopolymer systems is a raising trend. Nutraceuticals are highly suited for this purpose; however, their bioavailability is a great concern due to unstable and insoluble properties. Carriers play an indispensable role in controlled delivery and protection from external influences such as temperature, $\mathrm{pH}$, light, moisture, processing conditions during product developing as well as gastro-intestine transition (Nedovic et al., 2011; Ubbink \& Kruger, 2006). We recently ventured into developing carriers based on GRAS hydrocolloids that possess stable network structures (Janaswamy \& Youngren, 2012; Janaswamy, Gill, Campanella \& Pinal, 2013). Herein, we have used the $\mathrm{Na}$ and Ca salt forms of iota-carrageen as a suitable platform to examine the distinct roles of cations on the encapsulation efficiency and release nature of nutraceuticals employing eugenol as the model compound. The results point out the quintessential roles of nutraceutical-cation interactions in controlling the embedded amounts and subsequent release profiles. Additional research involving a wide variety of nutraceuticals and hydrocolloids need to be explored for gaining complete understanding of these novel

nutraceutical delivery systems. Our current focus is on cations $\mathrm{K}^{+}, \mathrm{Mg}^{2+}, \mathrm{Zn}^{2+}$ and $\mathrm{Fe}^{3+}$ as well as FDA approved solvents ethanol and propylene glycol, to name a few. The outcome indeed offers 
great opportunities for the design and development of more economical and efficient nutraceutical carriers leading to novel food supplements, functional foods and medicinal foods.

\section{Acknowledgements}

We thank Discovery Park Undergraduate Research Internship (DURI) program and Whistler Center for Carbohydrate Research (WCCR) for the support; FMC Corporation, USA for providing the iota-carrageenan sample; Dr. Yuan Yao for sharing the spectrophotometer facility; Dr. Bhavesh Patel for assisting with the DSC measurements, and Drs. Irina Kosheleva and Robert Henning for help during the synchrotron X-ray intensity data. Use of the Advanced Photon Source was supported by the U.S. Department of Energy, Basic Energy Sciences, Office of Science, under Contract No. DE-AC02-06CH11357. Use of the BioCARS Sector 14 was supported by the National Institutes of Health (NIH), National Center for Research Resources, under grant number RR007707. 


\section{References}

Acosta, E. (2009). Bioavailability of nanoparticles in nutrient and nutraceutical delivery. Current Opinion in Colloid \& Interface Science, 14, 3-15.

Bell, L. N. (2001). Stability testing of nutraceuticals and functional foods. In R. E. C. Wildman, Ed. Handbook of Nutraceuticals and Functional Foods (pp 501-516). CRC Press, New York.

Benencia, F., \& Courreges, M. C. (2000). In vitro and in vivo activity of eugenol on human herpesvirus. Phytotherapy Research, 14, 495-500.

Briozzo, J., Nunez, L., Chirife, J., Herszage, L., \& Daquino, M. (1989). Antimicrobial activity of clove oil dispersed in a concentrated sugar solution. Journal of Applied Bacteriology, 66, 69-75.

Chen, L., Remondetto, G. E. \& Subirade, M. (2006). Food protein-based materials as nutraceutical delivery systems. Trends in Food Science \& Technology, 17, 272-283.

Day, Li, Seymour, R. B., Pitts, K. F., Konczak, I., \& Lundin, L. (2009). Incorporation of functional ingredients into foods. Tends in Food Science \& Technology, 20, 388-395.

Dighe, V. V., Gursale, A. A., Sane, R. T., Menon, S., \& Patel, P. H. (2005). Quantitative determination of eugenol from cinnamomum tamala nees and eberm. Leaf powder and polyherbal formulation using reverse phase liquid chromatography. Chromatographia, $61,443-446$.

Goldberg, I. (1994). Ed. Functional foods. Chapman \& Hall, New York.

Jadhav, B. K., Khandelwal, K. R., Ketkar, A. R., \& Pisal, S. S. (2004). Formulation and evaluation of mucoadhesive tablets containing eugenol for the treatment of periodontal diseases. Drug Development and Industrial Pharmacy, 30, 195-203.

Jaganathan, S. K., Mazumdar, A., Mondhe,D., \& Mandal, M. (2011). Apoptotic effect of eugenol in human colon cancer cell lines. Cell Biology International, 35, 607-615.

Janaswamy, S., \& Chandrasekaran, R. (2001). Three-dimensional structure of the sodium salt of iota-carrageenan. Carbohydrate Research, 335, 181-194.

Janaswamy, S., \& Chandrasekaran, R. (2002). Effect of calcium ions on the organization of iota-carrageenan helices: an X-ray investigation. Carbohydrate Research, 337, 523-535.

Janaswamy, S., Gill, K. L., Campanella, O. H., \& Pinal, R. (2013). Organized polysaccharide fibers as stable drug carriers. Carbohydrate Polymers, 94, 209-215.

Janaswamy. S., \& Youngren, S. R. (2012). Hydrocolloid-based nutraceutical delivery systems. Food \& Function, 3, 503-507. 
Kim, S. S., Oh, O. J., Min, H. Y., Park, E. J., Kim, Y., Park, H. J., Han, Y. N., \& Lee, S. K. (2003). Eugenol suppresses cyclooxygenase-2 expression in lipopolysaccharide-stimulated mouse macrophage RAW264.7 cells. Life Sciences, 73, 337-348.

Langer, R. (1990). New methods of drug delivery. Science, 249, 1527-1533.

Langer, R., \& Tirrell, D. A. (2004). Designing materials for biology and medicine. Nature, 428, 487-492.

Lee, K. G., \& Shibamoto, T. (2001). Antioxidant property of aroma extract isolated from clove buds [Syzygium aromaticum (L.) Merr. et Perry]. Food Chemistry, 74, 443-448.

Nedovic, V., Kalusevic, A., Manojlovic, V., Levic, S., \& Bugarski, B. (2011). An overview of encapsulation technologies for food applications. Procedia Food Science, 1, 1806-1815.

Palaniappan, K., \& Holley, R. A. (2010). Use of natural antimicrobials to increase antibiotic susceptibility of drug resistant bacteria. International Journal of Food Microbiology, 140, 164-168.

Plapied, L., Duhem, N., des Rieux, A., \& Préat, V. (2011). Fate of polymeric nanocarriers for oral drug delivery. Current Opinion in Colloid \& Interface Science, 16, 228-237.

Raina, A. P., Kumar, A., \& Dutta, M. (2013). Chemical characterization of aroma compounds in essential oil isolated from "Holy Basil"' (Ocimumflaenui um L.) grown in India. Genetic Resources and Crop Evolution, 60, 1727-1735.

Rajkumar, G., Al-Khayat, H. A., Eakins, F., Knupp, C., \& Squire, J. M. (2007). The CCP13 FibreFix program suite: semi-automated analysis of diffraction patterns from non-crystalline materials. Journal of Applied Crystallography, 40, 178-184.

Shahidi, F. (2009). Nutraceuticals and functional foods: whole versus processed foods. Tends in Food Science \& Technology, 20, 376-387.

Stanley, N.F. (1990). Carrageenans. In: Harris, P. (Ed.) Food Gels (pp 79-119). London: Elsevier.

Ubbink, J., \& Krüger, J. (2006). Physical approaches for the delivery of active ingredients in foods. Tends in Food Science \& Technology, 17, 244-254.

Webb, G. P. (2006). Dietary Supplements and Functional Foods. Blackwell publishing Ltd., Oxford. 


\section{Figure captions}

Fig. 1. Schematic representation of hydrocolloid fiber preparation and encapsulation of nutraceuticals (NCs) in the fiber lattice. (a) A fiber puller containing fiber holder (white rectangle piece) and saturated salt solution (plastic wells) for maintaining the humidity. Glass plates from the top and bottom seal the fiber puller. (b) Placement of a drop of iota-carrageenan (IC) solution in between two glass rods. (c) Large scale preparation of IC fibers. Once the solution reaches semi-solid state, after around $3-4 \mathrm{hrs}$, each fiber holder will be loaded in the fiber puller and stretched. (d) The NC eugenol is encapsulated in a well-oriented and crystalline IC fiber. (e) The cross-section of the complex assembly containing ordered eugenol molecules (blue star) in the IC lattice, viewed down the helix-axis. The bigger stars with short and large arcs represent IC double helix of diameter $13.7 \AA \AA$. (f) Eugenol molecules are securely trapped in between a pair of IC helices, viewed normal to helix-axis and gain the required protection. The filled circles are cations (black), water molecules (green) and sulfate groups (red). (g) Adjacent IC helices are at $13.9 \AA$ separation in the presence of Na ions but are drawn closer to $13.6 \AA$ by the Ca ions, and thus the divalent ions result in much stronger network interactions.

Fig. 2. X-ray diffraction patterns from well-oriented and polycrystalline fibers of (a) Na iotacarrageenan and its complexes with eugenol prepared at (b) 4; (c) 25; (d) 35 and (e) $45^{\circ} \mathrm{C}$, and (f) Ca iota-carrageenan and its complexes prepared at (g) 4; (h) 25; (i) 35 and (j) $45^{\circ} \mathrm{C}$. The fibers are almost perpendicular to the incident X-ray beam. The first reflection on the meridian (an imaginary line in the north-south direction passing through the center) suggests the 3-fold helix. The circular ring on each pattern is from the calcite powder (3.035 characteristic spacing) for accurately estimating the detector to fiber distance.

Fig. 3. Intensity distribution on the first layer line in sodium iota-carrageenan (bottom most) and its complexes with eugenol prepared at $4,25,35$ and $45^{\circ} \mathrm{C}$ (from bottom to top).

Fig. 4. Modulated Differential Scanning Calorimetry (mDSC) profiles of iota-carrageenan (IC) fibers in the presence of (a) Na ions, and (b) Ca ions, (c) eugenol, and complexes of (d) Na IC- 
eugenol and (e) Ca IC-eugenol prepared at $4{ }^{\circ} \mathrm{C}$. The data confirm the thermal protection of eugenol by the IC network after encapsulation.

Fig. 5. The release rate of eugenol, measured at room temperature, from (a) the sodium (top) and calcium (bottom) iota-carrageenan (IC): eugenol complexes prepared at $25^{\circ} \mathrm{C}$, and (b) the complexes prepared at 4,35 and $45{ }^{\circ} \mathrm{C}$ (from bottom to top). The solid line represents Na IC complex and the broken line Ca IC. The eugenol molecules release instantaneously from the $\mathrm{Na}$ IC net but are held for about 18 mins or more by the Ca IC network. The inter-helical interactions in the presence of $\mathrm{Na}$ and $\mathrm{Ca}$ ions are responsible for the observed differences. 


\section{Graphical Abstract}

Hydrocolloid-based nutraceutical carrier systems maintain stable network structures leading to control release of nutraceuticals.

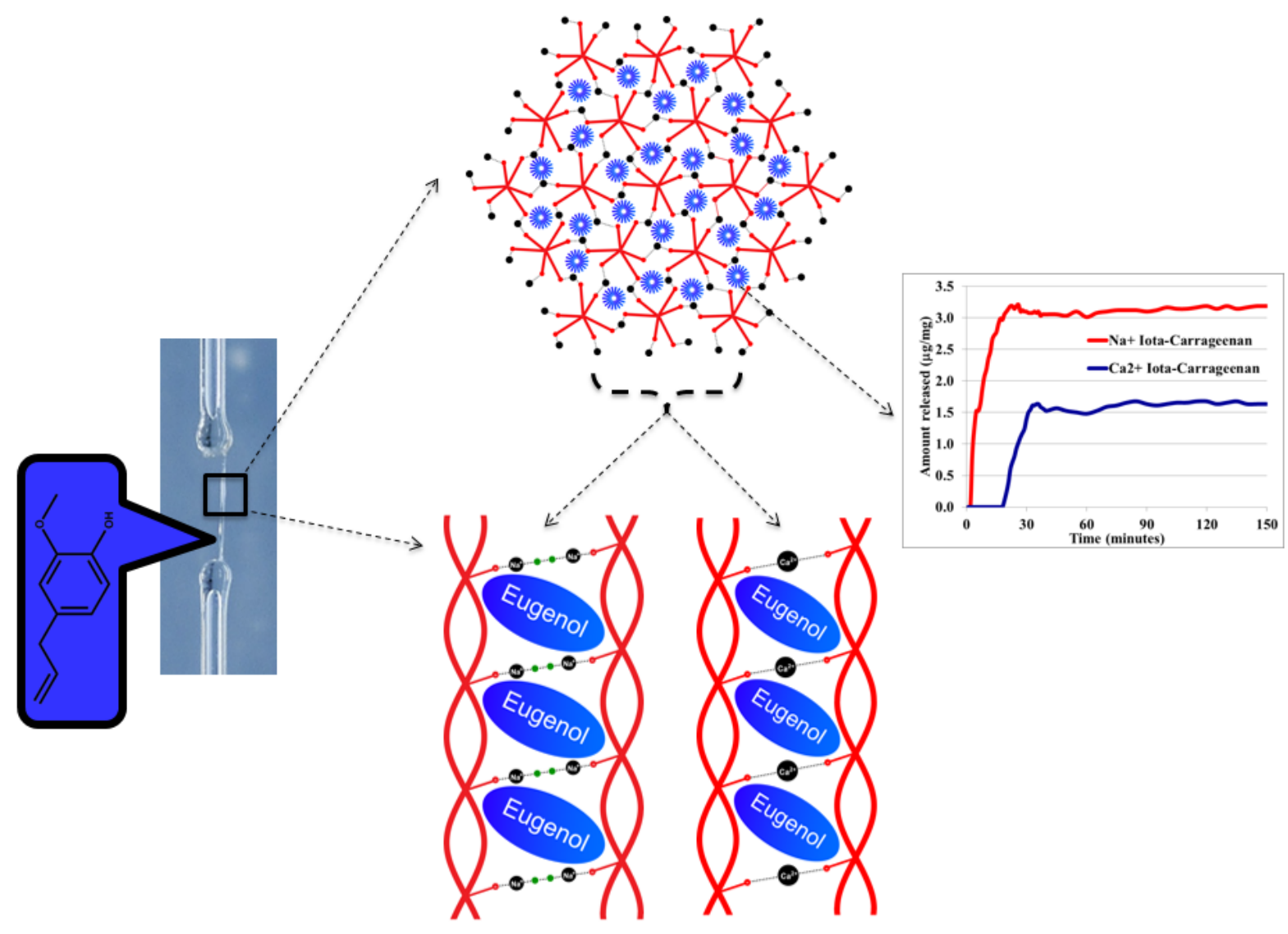




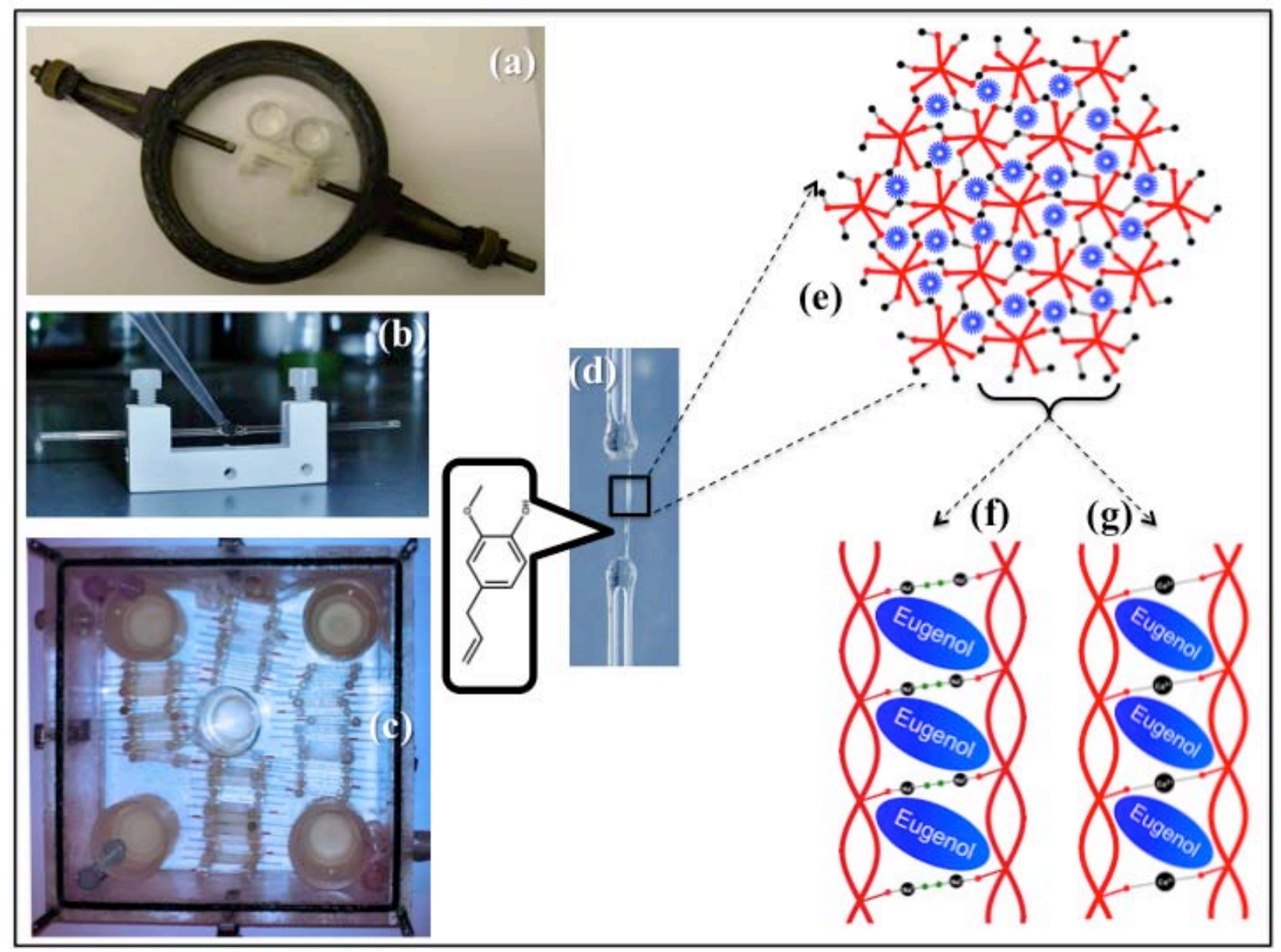

Fig. 1 


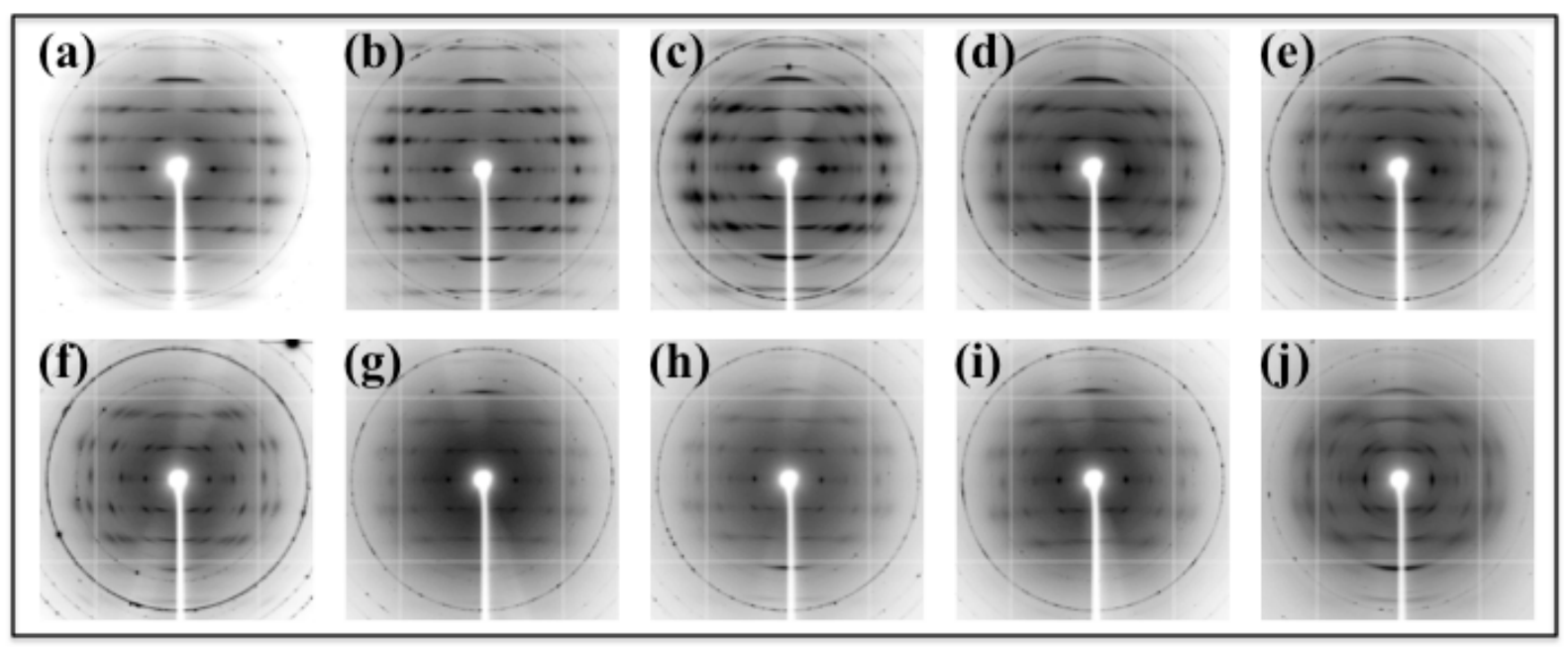

Fig. 2 


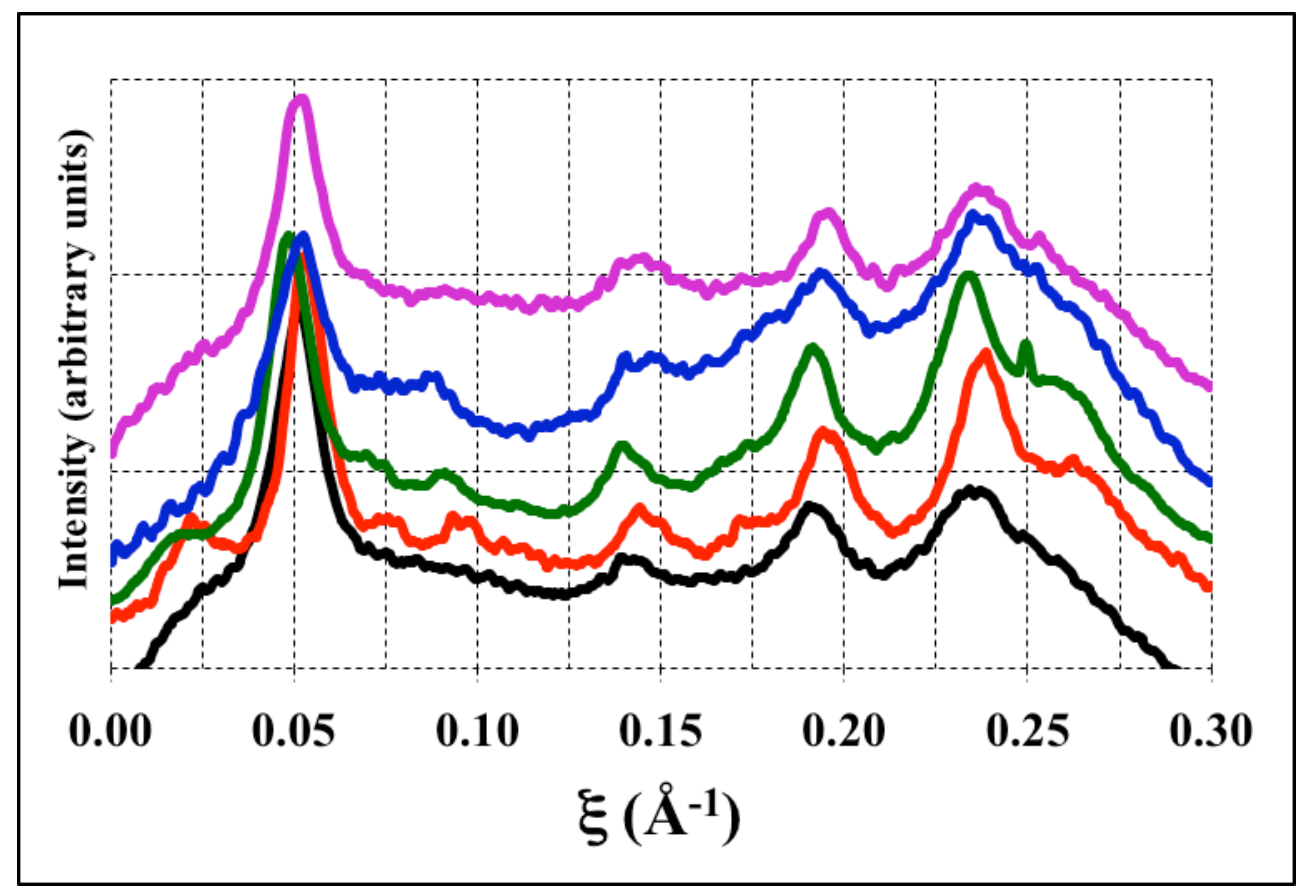

Fig. 3 


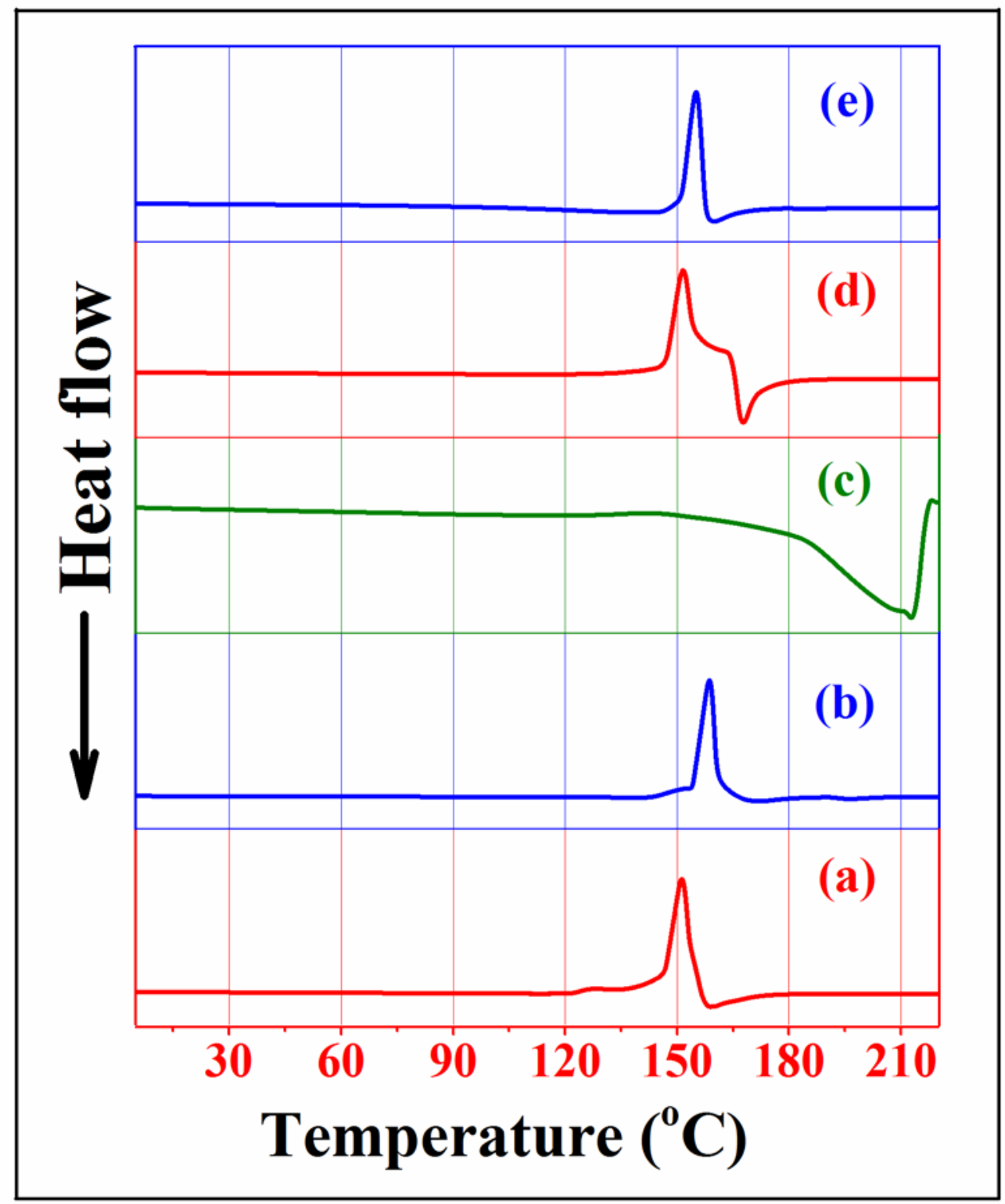

Fig. 4 


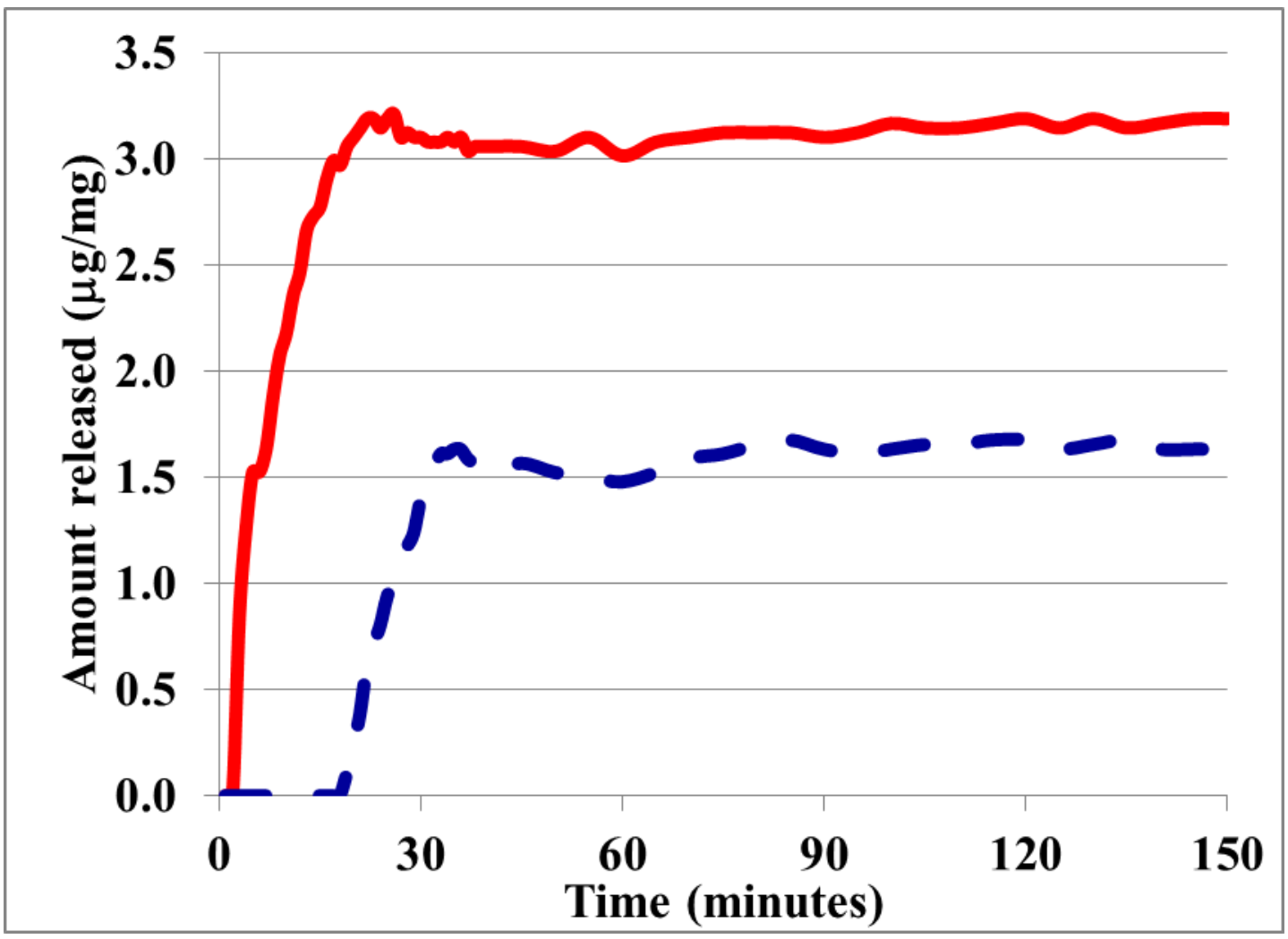

Fig. 5a

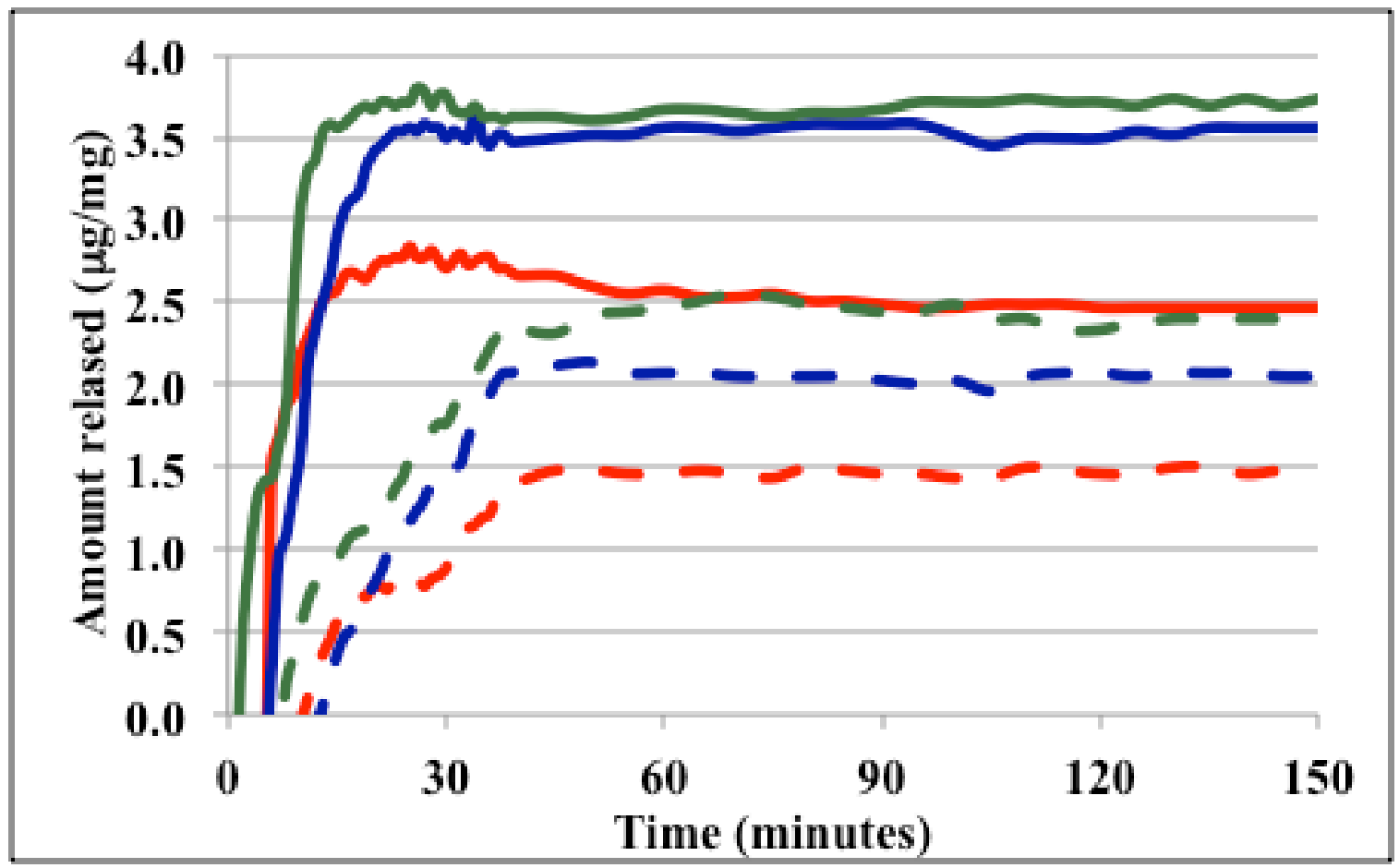

Fig. 5b 